\title{
Circulating bioactive sclerostin levels in an Austrian population-based cohort
}

\author{
Katharina Kerschan-Schindl (D) - Ursula Föger-Samwald • Andreas Gleiss - Stefan Kudlacek • \\ Jacqueline Wallwitz · Peter Pietschmann
}

Received: 16 September 2020 / Accepted: 13 January 2021 / Published online: 5 February 2021

(C) The Author(s) 2021

\begin{abstract}
Summary
Background Circulating serum sclerostin levels are supposed to give a good estimation of the levels of this negative regulator of bone mass within bone. Most studies evaluating total serum sclerostin found different levels in males compared to females and in older compared to younger subjects. Besides an ELISA detecting total sclerostin an ELISA determining bioactive sclerostin has been developed. The aim of this study was to investigate serum levels of bioactive sclerostin in an Austrian population-based cohort.

Methods We conducted a cross-sectional observational study in 235 healthy subjects. Using the bioactive ELISA assay (Biomedica) bioactive sclerostin levels were evaluated.

Results Serum levels of bioactive sclerostin were higher in men than in women $(24 \%)$. The levels correlated positively with age $(r=0.47)$. A positive
\end{abstract}

\footnotetext{
K. Kerschan-Schindl ( $₫)$

Department of Physical Medicine, Rehabilitation and Occupational Therapy, Medical University of Vienna, Waehringer Guertel 18-20, 1090 Vienna, Austria katharina.kerschan-schindl@meduniwien.ac.at

U. Föger-Samwald · P. Pietschmann

Department of Pathophysiology and Allergy Research, Center of Pathophysiology, Infectiology and Immunology, Medical University of Vienna, Vienna, Austria

A. Gleiss

Center of Medical Statistics, Informatics, and Intelligent Systems, Medical University of Vienna, Vienna, Austria

S. Kudlacek

Medizinische Abteilung, Krankenhaus Barmherzige Brüder, Vienna, Austria

\section{J. Wallwitz}

Department Pharmacology, Physiology and Microbiology, Division Pharmacology, Karl Landsteiner Privatuniversität für Gesundheitswissenschaften, Krems, Austria
}

correlation could also be detected with body mass index and bone mineral density.

Conclusion Using the ELISA detecting bioactive sclerostin our results are consistent with data in the literature obtained by different sclerostin assays. The determination of sclerostin concentrations in peripheral blood thus appears to be a robust parameter of bone metabolism.

Keywords Gender · Age - Body mass index · Bone turnover markers $\cdot$ Bone mineral density

\section{Introduction}

Diseases associated with high bone mass caused by mutation or deletion of the SOST gene led to the recognition of sclerostin $[1,2]$. The glycoprotein sclerostin consisting of about 200 amino acids is mainly expressed by osteocytes. As an antagonist of the osteoanabolic Wingless-type mouse mammary tumour virus integration site (Wnt) pathway sclerostin is an important regulator of bone metabolism. Binding to and thus inactivating the lipoprotein receptor-related protein 5 and 6 (LRP-5 and LRP-6) [3] leads to reduced osteoblast differentiation and activity. Thus, sclerostin inhibition was detected as a promising treatment option to preserve bone mass.

Circulating levels of sclerostin are supposed to reflect sclerostin levels in bone [4, 5]. Almost all investigations found higher sclerostin levels in males than in females [6-9]. Despite a few contradicting data [9, 10], most clinical studies detected an age-associated increase of serum levels of sclerostin [6, 11-14]. Although other companies also developed an assay, the majority of these studies used the quantitative sandwich enzyme-linked immunosorbent assay (ELISA) by Biomedica; however, by now the company has developed a new, a bioactive sclerostin ELISA. The previous 
assay used a polyclonal goat antibody and a monoclonal mouse antibody detecting various fragments of sclerostin proteins. The new assay is different. A recombinant monoclonal antibody binds to the second loop of the sclerostin core region capturing all sclerostin protein, which is able to bind to the LRP 5/6 complex of the Wnt signaling pathway thereby inhibiting bone formation. Thus, this ELISA captures all circulating proteins containing the free-receptor binding site, the bioactive sclerostin. The previous sclerostin ELISA as well as the bioactive sclerostin ELISA have been rigorously validated for clinical samples according to the Food and Drug Administration (FDA), the International Council for Harmonisation of Technical Requirements for Pharmaceuticals for $\mathrm{Hu}-$ man Use (ICH), and the European Medicines Agency (EMA) guidelines. The bioactive sclerostin ELISA has been shown to correlate with other sclerostin ELISAs, including the one previously developed by Biomedica. But there are some hints that in different types of diseases the bioactive sclerostin ELISA shows different results than the commonly used ELISAs [15]. Nevertheless, only two investigations used the bioactive sclerostin ELISA so far. Patients with spinal cord injuries [16] as well as patients with alcoholic liver disease [17] had lower levels than healthy controls. Thus, the aim of this cross-sectional study was to investigate serum levels of the physiologically relevant sclerostin, the bioactive sclerostin in a population-based cohort and to compare our data with data from the literature using different ELISAs. This study had explorative character. The working hypothesis was that bioactive sclerostin ELISA shows similar results as previous studies using a sclerostin ELISA.

\section{Material and methods}

\section{Participants}

All subjects included in this study were participants of a previously published investigation [18]. The former population register-based study evaluated several biochemical parameters and bone mineral density (BMD). A total of 235 subjects studied at 4 different Austrian outpatient bone clinics were randomly selected for this study. All subjects were healthy and did not take any medication affecting bone metabolism. Women who were on hormone replacement therapy (HRT) within the previous 5 years were excluded as well. The study protocol was approved by the ethics committee of the Krankenhaus Barmherzige Brüder, Vienna and all participants provided written informed consent after the procedure of the trial had been explained to them.

\section{Study procedures}

In all subjects, medical history was obtained and physical examination was performed. Their height was measured with a stadiometer and their weight was determined using a weight scale with a precision of $0.1 \mathrm{~kg}$. Body mass index (BMI) was calculated as weight $(\mathrm{kg})$ divided by the square of height $(\mathrm{m})$. After overnight fasting blood samples were collected in sterile chilled tubes by standard venepuncture technique. Blood was subsequently allowed to clot at room temperature. Aliquots of the centrifuged samples were stored at $-70^{\circ} \mathrm{C}$ until evaluation.

\section{Biochemistry}

Basic serum chemistry included evaluation of calcium, phosphate, creatinine, parathyroid hormone, 25-OH-vitamin D, and alkaline phosphatase. The bioactive form of sclerostin was measured with the quantitative sandwich ELISA kit from Biomedica (Bioactive Sclerostin ELISA BI 20472; Vienna, Austria). The intra-assay and interassay coefficients of variation $(\mathrm{CVs})$ were $<1 \%$ and $\leq 5 \%$, respectively. All samples were run in duplicate and in order to reduce inter-assay variation. They were analyzed in a single session. Bone turnover markers were evaluated as well. The bone resorption marker crosslinked-C-telopeptide of type I collagen (CTX; Cobas 8000 Roche Analyzer, Roche Diagnostics, Switzerland; detection limit: $0.5 \mathrm{ng} / \mathrm{mL}$; intra-assay coefficient of variation: $1.2-4.7 \%$, inter-assay coefficient of variation: $1.5-5.7 \%$ ) as well as the bone formation markers osteocalcin (Oc; Cobas 8000 Analyzer, Roche Diagnostics, Switzerland; detection limit: $0.01 \mathrm{ng} / \mathrm{mL}$; intra-assay coefficient of variation: $0.9-1.3 \%$, interassay coefficient of variation: $1.2-2.3 \%$ ) and $N$-terminal propeptide of type I collagen (P1NP; Cobas 8000 Roche Analyzer, Roche Diagnostics, Switzerland; detection limit: $5 \mathrm{ng} / \mathrm{mL}$; intra-assay coefficient of variation: $1.6-3.5 \%$; inter-assay coefficient of variation: $2.0-3.8 \%$ ) were determined. All analyses were conducted according to standard procedures.

\section{Bone mineral density measurement}

The BMD was measured at the lumbar spine and the proximal femur by dual energy x-ray absorptiometry (DXA) using DXA devices of two manufacturers (Lunar Corp., Madison, WI, USA and Hologic, Waltham, MA, USA). To ensure high quality of the data cross-calibration was performed with the use of the European Spine Phantom.

\section{Statistical analysis}

Continuous variables were described by median and quartiles due to non-normal distributions of most variables. Wilcoxon's rank-sum test was used for comparison between sexes. Serum levels of bioactive sclerostin were log-transformed for centre-adjusted comparison between sexes using an ANCOVA model. The resulting mean difference was back-transformed 


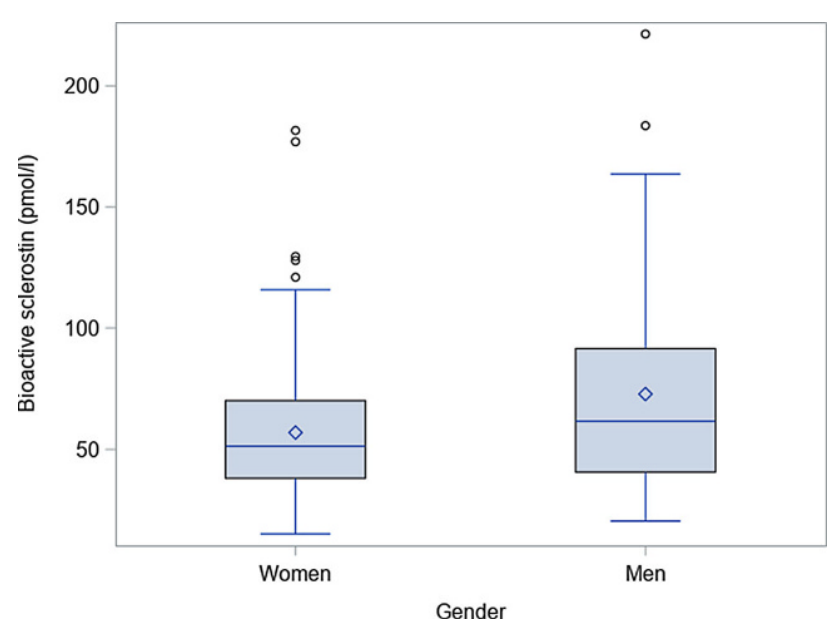

Fig. 1 Distribution of serum levels of bioactive sclerostin by gender. Data are given as boxplots

to the original scale resulting in a percent increase with $95 \%$ confidence interval (CI). Correlations were quantified using a Spearman correlation coefficient partialized for center. Two-sided $p$-values below 0.05 were considered to indicate statistical significance. All calculations were done using the statistical analysis software SAS 9.4 (SAS Inc., 2016).

\section{Results}

Characteristics and biochemical parameters of 175 women and 61 men are given in Table 1. Men were older and had a higher BMI than women. The BMD and most routine clinical chemistry parameters were similar in both groups. Serum levels of bioactive sclerostin were $24 \%$ higher in men than in women $(95 \%$ CI 9-41; $p=0.002$; Fig. 1). The levels correlated positively with age $(r=0.47 ; p<0.001 ;$ Fig. 2$)$. As shown in Table 2, a weak positive correlation was also detected with BMI $(\mathrm{r}=0.32 ; p<0.001)$ as well as the $\mathrm{T}$ score of the femoral neck $(\mathrm{r}=0.20 ; p=0.002)$.

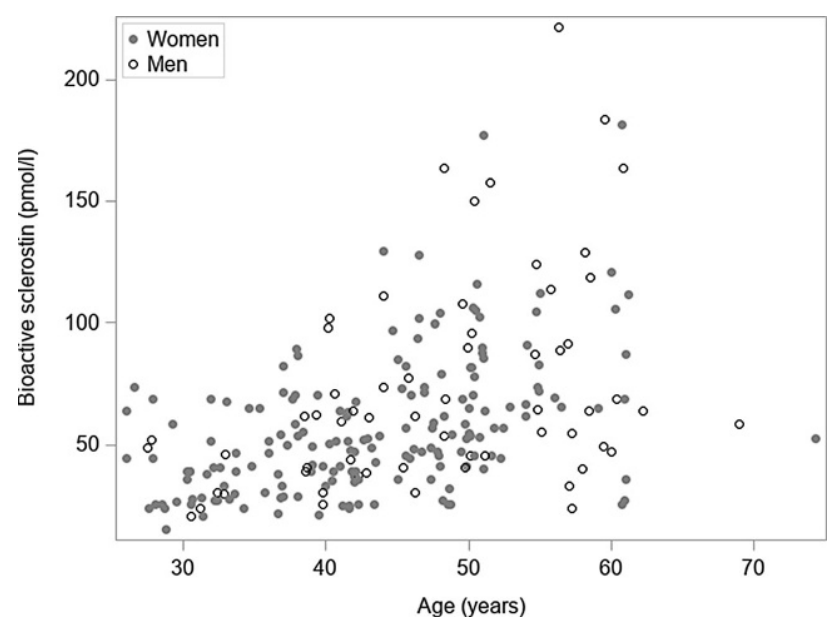

Fig. 2 Scatterplot showing the relation of serum levels of bioactive sclerostin and age
Table 1 Characteristics and biochemical parameters

\begin{tabular}{|c|c|c|c|}
\hline & Women $(N=175)$ & Men $(N=61)$ & $p$ \\
\hline Age (years) & $43[37,50]$ & $49[41 ; 56]$ & 0.001 \\
\hline BMI & $24[21 ; 27]$ & $26[24 ; 28]$ & $<0.001$ \\
\hline T score lumbar spine & $-0.4[-1.0 ; 0.6]$ & $0.0[-0.6 ; 1.0]$ & 0.053 \\
\hline T score femoral neck & $-0.3[-0.9 ; 0.4]$ & $-0.2[-0.7 ; 0.6]$ & 0.189 \\
\hline Calcium (mmol/l) & $2.4[2.3 ; 2.5]$ & $2.3[2.2 ; 2.4]$ & 0.011 \\
\hline Phosphate (mmol/l) & $1.2[1.1 ; 1.3]$ & $1.2[1.1 ; 1.4]$ & 0.931 \\
\hline Alkaline phosphatase (U/I) & $68[54 ; 84]$ & $74[62 ; 84]$ & 0.143 \\
\hline PTH (pg/ml) & $28[20 ; 38]$ & $27[19 ; 40]$ & 0.981 \\
\hline 25-OH-vitamin D (mmol/l) & $16[12 ; 25]$ & $19[14 ; 27]$ & 0.090 \\
\hline Creatinine (mg/dl) & $0.8[0.8 ; 0.9]$ & $1.0[0.9 ; 1.2]$ & $<0.001$ \\
\hline Bioactive sclerostin (pmol/l) & $51.3[38.1 ; 70.2]$ & $61.6[40.6 ; 91.6]$ & 0.002 \\
\hline CTX (ng/ml) & $0.15[0.09 ; 0.21]$ & $0.17[0.12 ; 0.27]$ & 0.013 \\
\hline Osteocalcin (ng/ml) & $20.9[16.1 ; 26.2]$ & 22.4 [19.2; 26.2] & 0.100 \\
\hline P1NP (ng/ml) & $36.7[28.3 ; 47.7]$ & 38.3 [30.5: 51.3] & 0.391 \\
\hline \multicolumn{4}{|c|}{$\begin{array}{l}\text { Data are expressed as median [IQR] } \\
N \text { number of subjects, BMI body mass index, } P T H \text { parathyroid hormone, } \\
C T X \text { cross-linked-C-telopeptide of type I collagen, } P 1 N P N \text {-terminal propep- } \\
\text { tide of type I collagen, IQR interquartile range }\end{array}$} \\
\hline
\end{tabular}

Table 2 Spearman correlation of bioactive sclerostin with age, body mass index and bone mineral density

\begin{tabular}{|l|l|l|}
\hline Age & $r$ & $p$ \\
\hline BMI & 0.47 & $<0.001$ \\
\hline T score lumbar spine & 0.32 & $<0.001$ \\
\hline T score hip & 0.1 & 0.059 \\
\hline BMI body mass index & 0.2 & 0.002 \\
\hline
\end{tabular}

Gender-specific correlation analyses corroborated the positive association of bioactive sclerostin with age (males: $r=0.38, p=0.003$; females: $r=0.47$, $p<0.001)$. In men $(\mathrm{r}=0.34 ; p<0.01)$ as well as in women $(\mathrm{r}=0.26 ; p<0.001)$ a relatively weak correlation with BMI was found. Concerning the correlation with the $\mathrm{T}$ score of the femoral neck, the correlation coefficients were $0.23(p=0.07)$ and $0.15(p<0.05)$ in men and women, respectively. Additionally, a weak negative association between bioactive sclerostin and osteocalcin levels was detected in males $(r=-0.29$ $p=0.0289$ ). No other associations between bioactive sclerostin and bone turnover markers could be established.

\section{Discussion}

This is the first study evaluating serum levels of bioactive sclerostin in a population-based cohort. It revealed higher levels in men than in women and a positive association with age, body mass index, and bone mineral density for males and females. A correlation with the bone formation marker osteocalcin could only be observed in males.

This study's results of higher levels in men than in women concur with the previous literature using different sclerostin ELISAs. Except for one study [11] 
which could not detect higher sclerostin levels in men after adjusting for several potential confounding factors, all other investigations [6-9, 19] found higher serum sclerostin levels in men than in women. That may be related to the following fact: osteocytes are the main source of sclerostin. Thus, circulating sclerostin levels may reflect the amount of osteocytes available. Of course, skeletal mass is larger in men than in women.

The age-associated increase of serum levels of bioactive sclerostin detected in this study is in line with most previous investigations, all of them using one of the former sclerostin ELISA assays. Except for an in vitro study which could not find age-related changes in sclerostin expression [20], most clinical studies detected an age-associated increase of serum levels of sclerostin [6, 11-14]; however, Dovjak et al. [7] and Moriwaki et al. [9] found an age-associated increase of serum sclerostin in men only. Study groups $[10,21,22]$ that investigated solely postmenopausal women could not detect an association between serum sclerostin and age; however, this study revealed a positive association of bioactive sclerostin with age in men and women.

The positive correlation between serum levels of bioactive sclerostin and BMI is in accordance with publications by Amrein et al. [11] and Kalem et al. [22]. Several other studies [7, 10, 14, 19] could not detect such an association. With 26-74 years this study had a remarkably similar age range as Amrein et al. [11]. The other studies investigated either subjects of higher age or very young and older subjects. Differences in age may be responsible for the presence or absence of a positive correlation. No previous study performed a correlation analysis for women and men separately. This study confirmed the positive association between serum levels of bioactive sclerostin and BMI for men as well as for women.

Since sclerostin is predominantly produced by osteocytes several studies investigated potential associations between serum sclerostin levels and bone mass. Not all $[12,22]$ but most studies detected a positive correlation between serum sclerostin and BMD [6, 8, $10,11,23$ ] and some also a correlation with better bone microarchitectural parameters or a lower fracture risk [6, 24]. This study's low correlation is in line with Amrein et al. [11]. Studies with a higher level of correlation investigated subjects of higher age [8, 23]. Mödder et al. [6] divided their participants into different age categories and detected that the age group 60 years plus showed a higher correlation between SOST and BMD than subjects between 40 and 59 years of age, whereas in the even younger age group no correlation was found. Thus, this study's correlation between serum levels of bioactive sclerostin and the $\mathrm{T}$ score of the femoral neck is in line with the previous literature. It is also in accordance with the finding that in osteoporotic bone characterized by a decrease of bone volume the expression of mRNA SOST is reduced [25]. So far only one study [6] performed a correlation analysis for both genders separately and found a positive correlation for men as well as for women above the age of 40 years for spine and total body BMD. In our study a positive association with the $\mathrm{T}$ score of the femoral neck was found in women and men; however, the level of significance could not be reached in men, a fact which most likely is due to the lower number of male participants.

In men, this study detected a weak negative correlation of bioactive sclerostin with osteocalcin $(-0.29$, $p=0.0289$ ). Several other studies investigated the association of sclerostin with bone formation markers, mostly P1NP. All of them [6, 7, 9-11, 14, 26] but one [21] also revealed a negative association of the same dimension.

Participants' vitamin D levels were well below the normal range; however, we do not believe that vitamin D deficiency influenced bioactive sclerostin levels. In kidney transplant recipients, a positive correlation between sclerostin and vitamin $\mathrm{D}$ has been found [27]. Nevertheless, in this study in line with a recent investigation of perimenopausal and postmenopausal women [28] no correlation between vitamin D and bioactive sclerostin levels could be detected. Median values of all bone turnover markers were within the normal range. Osteocalcin levels were similar to the study performed by Amrein et al. [11]. The fact that most women participating in this study were premenopausal may explain the relatively low CTX levels.

One limitation of this study is the cross-sectional design. Of course, it would have been even more informative to compare this bioactive sclerostin ELISA with different previous non-bioactive sclerostin ELISAs; however, limited sample volumes only permitted the determination of one instead of all sclerostin ELISAs. Thus, results of this study were compared with data of the previous literature using different sclerostin ELISAs.

\section{Conclusion}

Higher serum levels of bioactive sclerostin in men than in women and the positive association with age shown in this population-based cohort study are consistent with data in the literature obtained by different sclerostin assays. Thus, according to this study it is possible to use a sclerostin ELISA as well as a bioactive sclerostin ELISA. Consequently, the determination of sclerostin concentrations in peripheral blood appears to be a robust parameter of bone metabolism.

Acknowledgements We thank Biomedica Medizinprodukte $\mathrm{GmbH}$ for providing the bioactive sclerostin ELISA; BI-20472.

Funding Open Access funding provided by Medical University of Vienna 
Conflict of interest J. Wallwitz was employee of the Antibody Lab. Peter Pietschmann has received research support and/or honoraria from Amgen GmbH, Biomedica GmbH, DePuySynthes, Eli Lilly GmbH, Fresenius Kabi Austria, Meda Pharma/ Mylan GmbH, Shire Austria GmbH, TAmiRNAGmbH and UCB Pharma. K. Kerschan-Schindl, U. Föger-Samwald, A. Gleiss, and S. Kudlacek declare that they have no competing interests.

Open Access This article is licensed under a Creative Commons Attribution 4.0 International License, which permits use, sharing, adaptation, distribution and reproduction in any medium or format, as long as you give appropriate credit to the original author(s) and the source, provide a link to the Creative Commons licence, and indicate if changes were made. The images or other third party material in this article are included in the article's Creative Commons licence, unless indicated otherwise in a credit line to the material. If material is not included in the article's Creative Commons licence and your intended use is not permitted by statutory regulation or exceeds the permitted use, you will need to obtain permission directly from the copyright holder. To view a copy of this licence, visit http://creativecommons.org/licenses/by/4.0/.

\section{References}

1. Van Buchem FS, Hadders HN, Ubbens R. An uncommon familial systemic disease of the skeleton: hyperostosis corticalis generalisata familiaris. Acta Radiol. 1995;4:109-20.

2. Truswell AS. Osteopetrosis with syndactyly, a morphologic variant of Albers-Schönberg disease. J Bone Joint Surg. 1958;40:208-18.

3. Semenov M, Tamai K, HeX. SOST is a ligand for LRP5/ 6 and aWnt signaling inhibitor. J Biol Chem. 2005;260:770-5.

4. Drake MT, Srinivasan B, Mödder UI, Peterson JM, McCready LK, Riggs BL, et al. Effects of parathyroid hormone treatment on circulating sclerostin levels in postmenopausal women. J Clin Endocrinol Metab. 2010;95:5056-62.

5. Farr JN, Roforth MM, Fujita K, Nicks KM, Cunningham JM, Atkinson EJ, et al. Effects of age and estrogen on skeletal gene expression in humans as assessed by RNA sequencing. Plos One. 2015;10:e138347.

6. Mödder UI, Clowes JA, Hoey K, Peterson JM, McCready L, Oursler MJ, et al. Regulation of circulating sclerostin levels by sex steroids in women and in men. J Bone Miner Res. 2011;26:27-43.

7. Dovjak P, Dorfer S, Föger-Samwald U, Kudlacek S, Marculescu R, Pietschmann P. Serum levels of sclerostin and dickkopf1: effects of age, gender and fracture status. Gerontology. 2014;60:493-501.

8. Wanby P, Nobin R, Von SP, Brudin L, Carlsson M. Serum levels of the bone turnover markers dickkopf-1, sclerostin, osteoprotegerin, osteopontin, osteocalcin and 25-hydroxyviatimin D in Swedish 'geriatric patients aged 75 years or older with a fresh hip fracture in healthy controls. J Endocrinol Invest. 2016;39:855-63.

9. Moriwaki K, Matsumoto H, Tanishima S, Tanimura C, Osaki $\mathrm{M}$, Nagashima $\mathrm{H}$, et al. Association of serum boneand muscle-derived factors with age, sex, body composition, and physical function in community-dwelling middleaged and elderly adults: a cross-sectional study. BMC Musculoskelet Disord. 2019;20:276. https://doi.org/10.1186/ s12891-019-2650-9.

10. Xu XJ, Shen L, Yang YP, Lu FR, Zhu R, Shuai B, et al. Serum sclerostin levels associated with lumbar spine bone mineral density and bone turnover markers in patients with postmenopausal osteoporosis. Chin Med J.2013;126:2480-4.
11. Amrein K, Amrein S, Drexler C, Dimai HP, Dobnig H, Pfeifer K, et al. Sclerostin and its association with physical activity, age, gender, body composition, and bone mineral content in healthy adults. J Clin Endocrinol Metab. 2012;97:48-54.

12. Ardawi MS, Rouzi AA, Al-Sibiani SA, Al-Senani NS, Qari MH, Mousa SA. High sclerostin predicts the occurrence of osteoporotic fractures in postmenopausal women: the center of excellence for osteoporosis research study. J Bone Miner Res. 2012;27:2592-602.

13. Gunsser J, Hermann R, Roth A, Lupp A. Comprehensive assessment of tissue and serum parameters of bone metabolism in a series of orthopaedic patients. Plos One. 2019;14:e227133.

14. Xu Y, Gao C, He J, Gu W, Yi C, Chen B, et al. Sclerostin and its associations with bonemetabolism markers and sexhormones in healthy community-dwelling elderly individuals an adolescents. Front Cell Dev Biol. 2020;8:57. https://doi. org/10.3389/fcell.2020.00057.

15. Wallwitz J, Gadermaier E, Berg G, Himmler G. Comparison of the new bioactive Sclerostin ELISA with other commercially available Sclerostin immunoassays. (Abstract). Calcif Tissue Int. 2018;102:S1-S159. https://doi.org/10.1007/ s00223-018-0418-0.

16. Maimoun L, Bouallègue BF, Gelis A, Aouinti S, Mura T, PhilibertP, etal. Periostin and sclerostinlevelsinindividuals with spinal cord injury and their relationship with bone mass, bone turnover, fracture and osteoporosis status. Bone. 2019;127:612-9.

17. Wakolbinger R, Muschitz C, Wallwitz J, Bodlaj G, Feichtinger X, Schanda JE, et al. Serum levels of sclerostin reflect altered bone microarchitecture in patients with hepatic cirrhosis. Wien Klin Wochenschr. 2020;132:19-26.

18. Kudlacek S, Schneider B, Woloszczuk W, Pietschmann P, Willvonseder R, Austrian Study Group on Normative Values of Bone Metabolism. Serum levels of osteoprotegerin increase with age in a healthy adult population. Bone. 2003;32:681-6.

19. Bhattoa HP, Wamwaki J, Kalina E, Foldesi R, Balogh A, AntalSzalmas P. Serum sclerostin levels in healthy men over 50 yars of age. J Bone Miner Metab. 2013;31:579-84.

20. Rauner M, Sipos W, Pietschmann P. Age-dependent Wnt gene expression in bone and during the course of osteoblast differentiation. Age. 2008;30:273-82.

21. He J, Zhang H, Wang C, Zhang Z, Yue H, Hu W, et al. Associations of serum sclerostin and polymorphisms in the SOST gene with BMD and markers of bone metabolism in postmenopausal Chinese women. JClin Endocrinol Metab. 2014;99:E655-E73.

22. Kalem MN, Kalem Z, Akgun N, Bakirarar B. The relationship between postmenopausal women's sclerostin levels and their bone density, age, body mass index, hormonal status, and smoking and consumption of coffee and dairy products. Arch GynecolObstet. 2017;295:785-93.

23. GarneroP, Sornay-RenduE, MunozF, BorelO, ChapurlatRD. Association of serum sclerostin with bone mineral density, bone turnover, steroid and parathyroid hormones, and fracture risk in postmenopausal women: the OFELY study. Osteoporos Int. 2013;24:489-94.

24. Szulc P, Bertholon C, Borel O, Marchand F, Charpurlat P. Lower fracture risk in older men with higher sclerostin concentration: a prospective analysis from the MINOS study. JBone Miner Res. 2013;28:855-64.

25. Föger-Samwald U, Vekszler G, Hörz-Schuch E, Salem S, Wipperich M, Titschl P, et al. Molecular mechanisms of osteoporotic hip fractues in elderly women. Exp Gerontol. 2016;73:49-58. 
26. Amrein K, Dobnig H, Wagner D, Piswanger-Sölkner C, Pieber TR, PilzS, etal. Sclerostin in institutionalized elderly women associations with quantitative bone ultrasound, bone turnover, fractures, and mortality. J Am Geriatr Soc. 2014;62:1023-9.

27. Tartaglione L, Pasquali M, Rotondi S, Muci ML, Leonangeli C, Farcomeni A, et al. nteractions of sclerostin with FGF23, soluble klotho and vitamin D in renal transplantation. PLoS One. 2017;12:e178637.

28. Czajkowska M, Plinta R, Owczarek A, Olszanecka-Glinianowicz M, Skrzypulec-Plinta V. Circulating sclerostin levels in relation to nutritional status, sex hormones and selected bone turnover markers levels in peri- and postmenopausal women. Ginekol Pol. 2019;90:371-5.

Publisher's Note Springer Nature remains neutral with regard to jurisdictional claims in published maps and institutional affiliations. 